Artículos Originales / Original Articles

\title{
Cambios en la práctica laboral de los profesionales de enfermería por la pandemia SARS-CoV-2/COVID-19 en una ciudad de Colombia-
}

\author{
Changes in the labor practice of nursing professionals due to the SARS-CoV-2/ \\ COVID-19 pandemic in a city of Colombia
}

\author{
Claudia Alejandra Orgulloso Bautista ${ }^{1}$ (D) Leisly Beleño Pinedo ${ }^{2}$ (D) Rosa Isela Méndez Vergara ${ }^{3}$ (D) \\ Citación: Orgulloso CA, Beleño L, Méndez RI. Cambios en la práctica laboral de los profesionales de enfermería por la pandemia SARS-CoV-2/ \\ COVID-19 en ciudad una de Colombia. Ustasalud 2022;21(1): 25-31. DOI: https://doi.org/10.15332/us.v21i1.2603
}

Licencia Creative Commons

\section{(c) (1) (8) $\odot$} alterarlos, adicionalmente se debe reconocer la autoría de las personas que figuran en las publicaciones, pero estas no podrán ser comercializadas.

\section{Resumen}

Objetivo: Determinar los cambios en las prácticas laborales del profesional de enfermería a causa de la pandemia SARS-CoV-2/COVID-19. Materiales y Métodos: Estudio de corte transversal en una población de profesionales de enfermería que se encontraban laborando durante la pandemia en la ciudad de Cartagena, con una muestra total de 115 individuos a través de un muestreo de bola de nieve, a quienes se les aplicó una encuesta de manera virtual, con un análisis estadístico descriptivo. Resultados: La muestra estuvo conformada en su mayoría por mujeres, con un $82,6 \%$, en edades de 30 a 40 años con un $42,6 \%$, y el $72,2 \%$ tenían formación de pregrado. El 92,2\% de los profesionales de enfermería manifiestan que con la pandemia del SARS-CoV-2/COVID-19 se han presentado grandes cambios en su práctica laboral con el aumento de más actividades o tareas de las que se realizaban anteriormente, con la aplicación de nuevas estrategias en la atención y cuidados de los pacientes, con una alta percepción de riesgo en su lugar de trabajo para el contagio y dificultades económicas a nivel laboral por la pandemia y no consideran cambiar de perfil laboral u ocupacional por esta situación que está pasando. Conclusiones: La crisis sanitaria mundial actual, expone la necesidad que han tenido los profesionales de adaptar nuevas prácticas laborales y competencias a las nuevas demandas del mercado laboral.

Palabras clave: Enfermeros, perfil laboral, infecciones por coronavirus.

\section{Abstract}

Objective: To determine the changes in the work practices of the nursing professional due to the SARSCoV-2/COVID-19 pandemic. Materials and methods: Cross-sectional study in a population of nursing professionals who were working during the pandemic in the city of Cartagena, with a total sample of 115 individuals through snowball sampling, who were applied a survey virtually, a descriptive statistical analysis was performed. Results: The population consisted mostly of women with $82,6 \%$, in ages between 30 and 40 years with $42,6 \%$ and $72,2 \%$ had undergraduate training. The $92,2 \%$ of nursing professionals state that with the SARS-CoV-2/COVID-19 pandemic there have been great changes in their work practice with the increase in more activities or tasks than were previously carried out, with the application of new strategies in the care and care of patients, with a high perception of risk in their workplace for contagion and economic difficulties at the work level due to the pandemic and they do not consider changing their work or occupational profile due to this situation that is happening. Conclusions: The current global health crisis exposes the need for professionals to adapt new labor practices and skills to the new demands of the labor market.

Keywords: Nurses, job description, coronavirus infections.
1 Enfermera. Magíster en Epidemiología, Corporación Universitaria Rafael Núñez. Cartagena, Colombia.

2 Enfermera. Especialista en Cuidado al adulto y niño en estado crítico de salud, Magíster en Salud Sexual y Reproductiva, Corporación Universitaria Rafael Núñez. Cartagena, Colombia.

3 Estudiante de Enfermería, semillerista de la Corporación Universitaria Rafael Núñez, Cartagena, Colombia.

Autor de correspondencia:

Claudia Alejandra Orgulloso Bautista

\section{Correo electrónico:}

claudia.orgulloso@curnvirtual. edu.co 


\section{INTRODUCCIÓN}

En diciembre de 2019 se conocieron los primeros casos de un virus denominado SARS-CoV-2/COVID-19 procedente de Wuhan, provincia de Hubei en China. En el mes de enero la Organización Mundial de la Salud (OMS) alertó al mundo sobre una enfermedad respiratoria de carácter grave, de alto nivel de contagio y mortalidad que no se había visto en epidemias anteriores. Dado la acelerada replicación en más de 200 países la OMS declara la pandemia [1].

Por lo tanto, con el número cada vez mayor de casos infectados, los profesionales sanitarios que se encuentran en primera línea pueden estar bajo presión tanto física como psicológica [2]. Además, la pandemia producida por el SARS-CoV-2/COVID-19 ha puesto a prueba los sistemas de salud de los países en todo el mundo, las reacciones inmediatas y decisiones basadas en salud pública, estos cambios han llevado a conocer nuevos conceptos en la cotidianidad de las sociedades $\mathrm{y}$, además, el reforzamiento del autocuidado como medida de protección colectiva [3].

Igualmente, los profesionales del sector salud se han visto afectados debido a los diferentes cambios generados por la pandemia, desde afecciones en su salud mental y física, dado que se encuentran en la primera línea de defensa contra el virus, lo cual aumentó su carga de trabajo y jornada laboral. Desde esta perspectiva, la exposición de forma directa al propio patógeno constituye una presión contante en estos profesionales; afectando directamente el ejercicio de sus funciones, disminuyendo su desempeño laboral debido a las nuevas asignaciones a las que tuvieron que hacer frente [4]. Por lo anterior, pueden considerarse como aspectos que han transformado el entorno del personal de enfermería, generando grandes retos al profesional como el rediseñar estrategias para la adaptación a la nueva realidad, el fortalecimiento del trabajo en equipo y la calidad de atención a los pacientes [5].

Sin duda, la crisis sanitaria mundial actual expone la necesidad que han tenido los profesionales de adaptar sus perfiles y competencias a las nuevas demandas del mercado laboral. Por lo tanto, el presente estudio tiene como objetivo determinar los cambios en las prácticas laborales del profesional de enfermería a causa de la pandemia de SARS-CoV-2/COVID-19 en Cartagena.

\section{MATERIALES Y MÉTODOS}

Se realizó un estudio de corte transversal. La población fueron los profesionales de enfermería que se encontraban laborando en diferentes instituciones y servicios, como urgencias, hospitalización, cirugía, Unidad de Cuidados Intensivos y consulta externa durante la pandemia en la ciudad de Cartagena en el 2021. Para determinar el número de enfermeras participantes se realizó un muestreo no probabilístico por bola de nieve, debido a que no se tenía acceso a instituciones de salud para la toma directa de la información, definido por los protocolos instaurados por la situación actual. Se aplicaron como criterios de inclusión: profesionales de enfermería que estén laborando en instituciones prestadoras de servicios de salud y educación durante la pandemia por SARS-CoV-2/COVID-19.

Para la recolección de datos se creó una encuesta en formato virtual, la cual fue validada por el equipo de docentes del Comité de Investigación general, dadas las condiciones de la pandemia la encuesta se creó y aplicó con la herramienta Google Forms. Dicho instrumento constó de 32 preguntas, que se subdividieron en 3 componentes: I. Datos sociodemográficos, II. Condiciones laborales y III. Cambios en la práctica laboral. Se aplicó inicialmente a los profesionales de enfermería conocidos y egresados de la institución por medio de difusión del enlace de la encuesta por correo o por celular y a través de ellos difundir a otras personas.

\section{Análisis estadístico}

Se hizo un análisis descriptivo. Las variables cualitativas (sociodemográficas, condiciones de trabajo y práctica laboral) fueron presentadas como valores absolutos y relativos, los datos fueron analizados en el software Stata versión 13.

\section{Consideraciones éticas}

La presente investigación toma en consideración la Declaración de Helsinki, con relación al respeto por la libertad del individuo en la participación del estudio y la Resolución 008430 de 1993 de la República de Colombia, que representa una investigación sin riesgo, en la que se incluyeron técnicas y métodos de investigación documental retrospectivos de los individuos 
que participaron en el estudio. Así mismo, este estudio contó con la autorización y aval ético ante el Comité de Ética de la institución patrocinadora.

\section{RESULTADOS}

De acuerdo con las características sociodemográficas, la muestra estuvo conformada por 115 profesionales de enfermería que se encontraban trabajando en pandemia, en la cual un $82,6 \%$ eran mujeres, el $42,6 \%$ tenían edades entre 30 y 40 años, el 39,1\% eran solteros, y su mayor nivel de educación alcanzado era pregrado con un $72,2 \%$ (Tabla 1 ).

Tabla 1. Características sociodemográficas de los participantes del estudio

\begin{tabular}{lcc}
\hline \multicolumn{1}{c}{ Variables } & n & $\%$ \\
\hline Sexo & & \\
\hline Mujer & 95 & 82,6 \\
\hline Hombre & 20 & 17,4 \\
\hline Edad (años) & & \\
\hline Menos de 30 & 42 & 36,5 \\
\hline 30-40 & 49 & 42,6 \\
\hline 41-60 & 24 & 20,9 \\
\hline Estado Civil & & \\
\hline Soltero & 45 & 39,1 \\
\hline Casado & 34 & 29,6 \\
\hline Unión libre & 31 & 27 \\
\hline Divorciado & 3 & 2,6 \\
\hline Separado & 2 & 1,7 \\
\hline Personas a cargo & & \\
\hline Menos de 2 & 88 & 76,1 \\
\hline 3 - 5 & 25 & 21,8 \\
\hline Más de 5 & 2 & 1,7 \\
\hline Nivel de educación & & \\
\hline Pregrado & 83 & \\
\hline Especialización & 24 & \\
\hline Maestría & & \\
\hline Doctorado & & \\
\hline & & \\
\hline
\end{tabular}

Fuente: Autores.

Con respecto a las condiciones de la práctica laboral, la mayoría pertenece a instituciones privadas $(70,4 \%)$, con un contrato de tipo indefinido en $47,8 \%$ de ellos, con una experiencia laboral entre los 6 a 15 años con un $36,5 \%$, trabajando en una sola empresa y con 48 horas laborales o más en un 45,2\% (Tabla 2). Igualmente, el 92,2\% de los profesionales de enfermería manifiestan que con la pandemia del SARS-CoV-2/ COVID-19 se han presentado grandes cambios en su práctica laboral con el aumento de más actividades o tareas de las que se realizaban anteriormente (Figura 1).

Tabla 2. Condiciones laborales de profesionales de enfermería participantes en el estudio en Cartagena durante la pandemia SARS-CoV-2/COVID-19

\begin{tabular}{lcc}
\hline \multicolumn{1}{c}{ Variables } & $\mathbf{n}$ & $\%$ \\
\hline \multicolumn{2}{l}{ Tipo de institución donde labora } & \\
\hline Privada & 81 & 70,4 \\
\hline Pública & 34 & 29,6 \\
\hline Tipo de contrato & \\
\hline $\begin{array}{l}\text { De servicios } \\
\text { temporales }\end{array}$ & 35 & 30,4 \\
\hline Fijo & 25 & 21,8 \\
\hline Indefinido & 55 & 47,8 \\
\hline Experiencia laboral (años) & 15 & 13,1 \\
\hline Menos de 1 & 34 & 29,6 \\
\hline 1 a 5 & 42 & 36,5 \\
\hline 6 a 15 & 24 & 20,8 \\
\hline Más de 15 & 106 & 92,2 \\
\hline Número de empresas donde labora & 5,2 \\
\hline 1 & 6 & 2,6 \\
\hline Más de 1 & 3 & 18,3 \\
\hline Más de 3 & 52 & \\
\hline Horas laborales (horas) & & \\
\hline 24 o menos & & \\
\hline 36 a 48 & & \\
\hline 48 o más & & \\
\hline
\end{tabular}

Fuente: Autores.

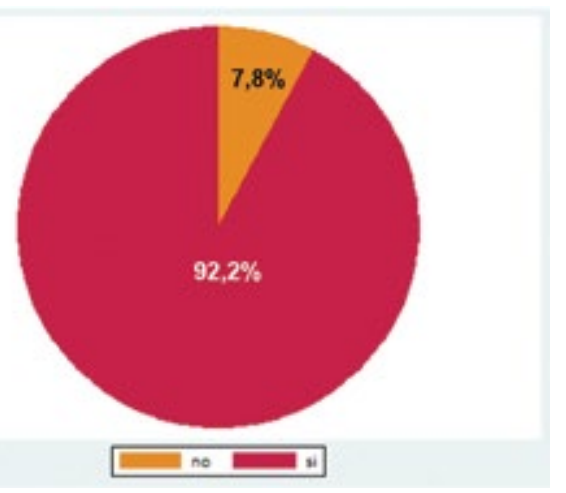

Figura 1. Proporción de cambios en la práctica laboral de los profesionales de enfermería por la pandemia SARS-CoV-2/COVID-19. 
Igualmente, de acuerdo con los cambios de aspectos laborales en el profesional de enfermería por el SARSCoV-2/COVID-19, refiriendo el 70,4\% que durante esta pandemia se ha tenido que aplicar nuevas estrategias en la atención y cuidados de los pacientes, principalmente estrategias de uso de elementos de protección personal como medidas de protección; tanto para ellos como para los pacientes, en un $43,5 \%$. Además, el $72,2 \%$ tiene una alta percepción de riesgo en su lugar de trabajo para el contagio. Sin embargo, un $68,7 \%$ manifestó que están profesionalmente capacitados para la atención de esta situación. Finalmente, el $41,8 \%$ presentaron principalmente dificultades económicas a nivel laboral por la pandemia y no consideran cambiar de perfil laboral u ocupacional por la situación en un 65,2\% (Tabla 3).

\section{DISCUSIÓN}

Este estudio descriptivo presenta resultados significativos donde se observó que los profesionales de enfermería en su mayoría trabajan en instituciones privadas, con contratos indefinidos, con experiencia laboral mayor a los seis años y con horas laborales de igual o mayor a 48 horas. Además, refirieron grandes cambios por la pandemia, en especial es sus planes de cuidados a los pacientes y sin olvidar el alto riesgo de contagio por su cercanía a los pacientes a pesar de estar capacitados.

Teniendo en cuenta, que el nuevo coronavirus ha sido definido por la OMS como: emergencia en salud pública de trascendencia mundial. Además, es posible afirmar que la población a nivel mundial ha experimentado distintas situaciones que han transformado la rutina diaria de cada hogar y trabajador [6]. Donde, de acuerdo con lo reportado en China los profesionales sanitarios, en especial los enfermeros que colaboran en la pandemia representan el $68 \%$ (28.600) y han sido considerados la principal mano de obra en el control de esta [7]. Es entonces donde el personal de enfermería se marca por cambios rápidos en sus prácticas laborales, aceptación del cumplimiento de otras funciones, la terminación de contratos e ingreso de nuevo personal en lugares donde la demanda de pacientes se considera alta. Por lo tanto, se realiza el presente estudio que tuvo como objetivo el determinar los cambios en los perfiles y las prácticas laborales del profesional de enfermería a causa de la pandemia de SARS-CoV-2/COVID-19.
Tabla 3. Cambios de aspectos laborales en el profesional de enfermería por la pandemia SARS-CoV-2/COVID-19

\begin{tabular}{lcc}
\hline Variables & $\mathrm{n}$ & $\%$ \\
\hline \multicolumn{4}{l}{ Aplicación de nuevas estrategias para la atención } & \\
\hline Sí & 81 & 70,4 \\
\hline No & 22 & 19,1 \\
\hline Tal vez & 12 & 10,5 \\
\hline Estrategias aplicadas para la atención & & \\
\hline Horarios de atención & 12 & 10,5 \\
\hline Modelo de atención & 29 & 25,2 \\
\hline Elementos de protección personal & 50 & 43,5 \\
\hline Otros & 24 & 20,8 \\
\hline Dificultades laborales presentadas por la pandemia & \\
\hline Económico & 48 & 41,8 \\
\hline Salud & 22 & 19,1 \\
\hline Ninguno & 41 & 35,7 \\
\hline Otro & 4 & 3,4 \\
\hline Percencion del riesgo &
\end{tabular}

Percepción del riesgo de contraer SARS-CoV-2

\begin{tabular}{lcc}
\hline Alto & 83 & 72,2 \\
\hline Medio & 19 & 16,5 \\
\hline Bajo & 13 & 11,3 \\
\hline Asignación de tarea diferente a sus funciones & \\
\hline Sí & 51 & 44,3 \\
\hline No & 64 & 55,7 \\
\hline
\end{tabular}

Jornada laboral diaria con la pandemia

\begin{tabular}{lll}
\hline Aumentó & 78 & 67,8 \\
\hline Disminuyó & 15 & 13,1 \\
\hline Está igual & 22 & 19,1 \\
\hline
\end{tabular}

Competencias profesionales necesarias para la atención

\begin{tabular}{lll}
\hline Sí & 79 & 68,7 \\
\hline No & 36 & 31,3 \\
\hline
\end{tabular}

Consideración de cambiar perfil laboral $\mathrm{u}$ ocupacional por la pandemia

\begin{tabular}{lll}
\hline Sí & 40 & 34,8 \\
\hline No & 75 & 65,2 \\
\hline
\end{tabular}

Fuente: Autores. 
En consecuencia, la pandemia ha presentado la relevancia de disponer la cantidad adecuada de profesionales de salud de acuerdo con la necesidad de cuidado en cada zona geográfica; para que se brinden buenas condiciones de trabajo (equipos de protección personal, nuevos cuidados e impulso al trabajo en equipo), en las que los profesionales de enfermería sean considerados como la primera línea de atención y los cuidados se establezcan de acuerdo con los roles de su práctica laboral [8]. Sin embargo, en el país hasta la fecha no se cuenta con una política pública adaptada para los cambios ocasionados a través de la pandemia y sus municipios no cuentan con un plan formulado de reducción de las carencias que tiene su población para que tienda hacia el abordaje integral en oferta y consumo [9]. Ahora bien; con los presentes resultados del estudio, un 92,2\% de los profesionales de enfermería de Cartagena manifestaron que han presentado cambios en la práctica laboral por la pandemia, que se han obligado a la aplicación de nuevas estrategias de atención entre las que se encuentran el uso de elementos de protección personal y modelos de atención.

Por otra parte, en un estudio en China se observó que la tasa de ansiedad del personal de salud fue del $23,04 \%$, mayor en mujeres que en hombres y mayor entre las enfermeras que entre los médicos [10]. Resultados similares a otro estudio que reporta un nivel de carga de trabajo mental en las enfermeras con altas autoevaluaciones de su desempeño bajo cargas de tareas extremadamente intensivas. Además, se identificaron en un $8,6 \%$ con "baja carga de trabajo y baja autoevaluación", un 35,3\% de "carga de trabajo y autoevaluación medias" y 56,1\% con una "carga de trabajo y autoevaluación altas" [11]. Sin embargo, en el caso del presente estudio el personal refirió que la dificultad que más presentaron fue a nivel económico y solo un 19,1\% manifestó dificultades a nivel de salud.

Además, de acuerdo con otros estudios se ha podido documentar que, durante esta pandemia, la carga laboral se ha aumentado significativamente y esta se encuentra relacionada con el gran volumen de pacientes con signos y síntomas, escasez de personal, lo que ha generado fatiga en los profesionales de la salud [12]. Igualmente, Orozco y otros indican que las exigencias y la intensificación del ritmo de trabajo aumentan la sobrecarga laboral, por la atención a un gran número de pacientes y la realización de funciones ajenas al ejercicio del cuidado directo [13], y algunos incluso se movilizaron más allá de sus horarios de trabajo, para prestar la atención al llamado del deber y proporcionar la ayuda necesaria [14]. Resultados similares al presente estudio donde el $67,8 \%$ de los profesionales de enfermería confirman que su carga laboral se ha aumentado, debido a la presencia de muchos pacientes con sospecha o confirmación del virus en las instituciones de salud.

Igualmente, los profesionales de enfermería llamados a atender en primera línea a las personas que presentan SARS-CoV-2/COVID-19 son preparados de manera científica, con una visión integral que los hace líderes para afrontar todas las situaciones con ética, desde diferentes puntos de vista: administrativo, investigativo, docente, asistencial en el ámbito clínico y comunitario. Con el conocimiento pertinente asumen su responsabilidad de cuidado en la situación real que vive la población [15]. Situación confirmada por los participantes de la investigación donde el $68,7 \%$ refirió que tiene las competencias profesionales necesarias para la atención de estos pacientes y no consideran cambiar su perfil laboral u ocupacional por la pandemia. Sin embargo, si bien el personal de enfermería está orientado a brindar una atención integral en salud por parte de los profesionales, donde se hace necesario aplicar las competencias o capacitaciones adquiridas, dichas competencias deben ser mejoradas para estar preparados y enfrentar esta crisis que se está presentando en la actualidad [16].

Dentro de las limitaciones del estudio, se presentó dificultad al momento de la recolección de los datos, debido a no poder acceder directamente a las instituciones de salud y, por lo tanto, fue muy poca la población abordada en el estudio, lo que introduce al sesgo de selección. Por otro lado, se pudo presentar el sesgo de información no diferencial, relacionado con la medición por medio del auto reporte, lo cual llevaría a una disminución de la magnitud de esta. Además, es importante tener en cuenta variables de la caracterización del servicio donde laboran los profesionales de enfermería que tienen relación con la carga de pacientes. Sin embargo, es importante resaltar las fortalezas de la presente investigación; se observó una percepción de que han ocurrido muchos cambios en el aspecto laboral 
del profesional de enfermería debido a la pandemia del SARS-CoV-2/COVID-19, se han incrementado sus horas laborales (carga laboral), y la necesidad de crear nuevas estrategias para el cuidado de los pacientes no sin olvidar el riesgo que estos tienen que asumir por ser la primera línea de atención. Por lo tanto, se recomienda más investigación, donde no solo se tengan en cuenta los aspectos laborales, sino también aspectos que tiene que ver con las posibles consecuencias que estos cambios han generado en la salud y a nivel social. Además, es importante el apoyo que puede brindar el personal de enfermería en este escenario, es fundamental para mitigar las dificultades que presentan los servicios de salud, así como para favorecer las medidas de protección personal y la abogacía en beneficio del ser humano [8]. Todo esto con el fin de proporcionar información para la creación de estrategias que no solo protejan al personal en aspectos de elementos de protección, sino también en lo que está a su alrededor, como personas que tienen el cuidado de otros.

En la relevancia de los resultados obtenidos es importante considerar promover los espacios investigativos a través de la realización de nuevos estudios prospectivos que incluyan la medición de otras variables, como las condiciones laborales, los servicios de atención, para así poder tener un análisis más completo y poder planear mejor las intervenciones, estrategias, programas y políticas en salud pública. Igualmente, continuar con la creación de conocimiento y con su apropiación social, para reconocer la ardua labor del personal de enfermería y minimizar los retos a los que han tenido que enfrentarse durante la pandemia de SARS-CoV-2/COVID-19, además de velar porque se disminuyan los obstáculos que ellos tienen para cumplir con su trabajo.

\section{REFERENCIAS}

[1] Kohen J. El trabajo y la salud en tiempos de COVID-19. Mientras continúa la pandemia. 2020. [Citado 15 octubre 2020]. URL disponible en: https://rephip.unr.edu.ar/ handle/2133/18821

[2] Lai J, Ma S, Wang Y, Cai Z, Hu J, Wei N, et al. Factors associated with mental health outcomes among health care workers exposed to coronavirus disease 2019. Rev JAMA Network Open. 2020;3(3): e203976. URL disponible en: https://doi.org/10.1001/jaman etworkopen.2020.3976

[3] Ministerio de Salud de Colombia. El reto del COVID-19 para los sistemas de salud en el mundo [Internet].
Minsalud.gov.co; 2020. [Citado 15 octubre 2020]. URL disponible en: https://www.minsalud.gov.co/Paginas/ El-reto-del-covid-19-para-los-sistemas-de-salud-en-elmundo.aspx

[4] García JJ, Gómez J, Martín J, Fagundo J, Ayuso D, Martínez JR, Ruiz C. Impacto del SARS-CoV-2 (COVID-19) en la salud mental de los profesionales sanitarios: una revisión sistemática. Rev Esp Salud Pública. 2020;94: 23 de julio e202007088. URL disponible en: https://rua. ua.es/dspace/bitstream/10045/108234/1/Garcia-Iglesias_etal_2020_RevEspSaludPublica.pdf

[5] Velásquez CM. 2020, el año de la enfermería en Colombia y sus retos ante la pandemia por COVID-19. Revista Colombiana de Enfermería 2020;19(2):1-4. DOI: https:// doi.org/10.18270/rce.v19i2.3139.

[6] Ministerio de Salud de Colombia. ABECÉ. Nuevo Coronavirus (COVID-19) de China [Internet]. Minsalud.gov. co. [Citado 23 abril 2021]. URL disponible en: https:// www.minsalud.gov.co/sites/rid/Lists/BibliotecaDigital/ RIDE/VS/PP/ET/abece-coronavirus.pdf

[7] National Health Commission of the People's Republic of China. Joint prevention and control mechanism press conference on introduction of new coronavirus pneumonia treatment and patient rehabilitation by State Council on february 29, 2020 [Citado 20 mayo 2021].

[8] Cassiani SHB, Munar EF, Umpiérrez A, Peduzzi M, Leija C. La situación de la enfermería en el mundo y la región de las Américas en tiempos de la pandemia de COVID-19. Rev Panam Salud Publica, 2020;44:e64. DOI: https://doi.org/10.26633/RPSP.2020.64

[9] Guan Wj, Ni Zy, Hu Y, Liang Wh, Ou Cq, He Jx, et al. Clinical characteristics of coronavirus disease 2019 in China. New England journal of medicine, 2020;382(18):17081720. DOI: https://doi.org/10.1056/NEJMoa2002032.

[10] López MJ, Majo HR. Situación de enfermería en tiempos de COVID-19: Una mirada panorámica. Journal of America health, 2020;3(3):48-58. DOI: https://doi. org/10.37958/jah.v3i3.50.

[11] Shan Y, Shang J, Yan Y, Lu G, Hu D, Ye X. Mental workload of frontline nurses aiding in the COVID-19 pandemic: A latent profile analysis. J Adv Nurs. 2021;77(5):2374-2385. DOI: $10.1111 /$ jan.14769.

[12] Núñez JM. COVID-19 por SARS-Cov2 también ha afectado a la Educación Médica. Educ Med. 2020;21(4):261264. DOI: https://doi.org/10.1016/j.edumed.2020.06.001

[13] Orozco MM, Zuluaga YC, Pulido G. Factores de riesgo psicosocial que afectan a los profesionales en enfermería. Revista Colombiana de Enfermería, 2019;18(1):1-16. DOI: https://doi.org/10.18270/rce.v18i1.2308

[14] Sohrabi C, Alsafi Z, O’Neill N, Khan M, Kerwan A, Al-Jabir A, Agha R. World Health Organization declares global 
emergency: A review of the 2019 novel coronavirus (COVID-19). International Journal of Surgery, 2020;76,71-76. DOI: https://doi.org/ 10.1016/j.ijsu.2020.02.034.

[15] Morales BV, Palencia JJ. Reflexiones del cuidado enfermero en tiempos de COVID-19. Enfermería Investiga, 2020;5 (3):71-78. URL disponible en: https://revistas.uta. edu.ec/erevista/index.php/enfi/article/view/914
[16] Pérez R, Doubova S. Los retos del personal de salud ante la pandemia de COVID-19: pandemónium, precariedad y paranoia. BID mejorandovidas. 2018 [citado el 23 de septiembre de 2020]. URL disponible en: https://blogs. iadb.org/salud/es/desafios-personal-salud-coronavirus/

\section{Correo de autores}

Claudia Alejandra Orgulloso Bautista: claudia.orgulloso@curnvirtual.edu.co

Leisly Beleño Pinedo: leisly.beleno@curnvirtual.edu.co

Rosa Isela Méndez Vergara: rmendezv6@curnvirtual.edu.co 